\title{
Our Experience with Embryonal Rhabdomyosarcoma Presenting as Aural Polyp
}

\author{
Anoop Attakkil, Vandana Thorawade, Mohan Jagade, Rajesh Kar, Dnyaneswar Rohe, \\ Reshma Hanowate, Devkumar Rangaraja, Kartik Parelkar \\ Department of ENT, Grant Medical College \& Sir J.J. Hospital, Mumbai, India \\ Email: fasttrack2317@gmail.com
}

Received 1 November 2014; revised 2 December 2014; accepted 17 December 2014

Copyright (C) 2015 by authors and Scientific Research Publishing Inc.

This work is licensed under the Creative Commons Attribution International License (CC BY). http://creativecommons.org/licenses/by/4.0/

(c) (i) Open Access

\begin{abstract}
Aural polyps are a common clinical entity encountered by otorhinolaryngologist in daily practice. Polyps are frequently seen in paediatric patients, usually inflammatory in nature. In children rhabdomyosarcomas (RMS) can mimic all the cinical features of chronic suppurative otitis media which usually present as external auditory canal mass or polyp. Here we present a case where a male child presented with recurrence of polyp in left ear which was finally diagnosed as embryonal rhabdomyosarcoma which is a rare and invariably fatal disease in children. Through this article we intend to highlight the failures and delay committed in attaining diagnosis in this patient in spite of multispecialty evaluation involving repeated imaging and histopathological correlation. Our experience with embryonal rhabdomyosarcoma throws light on the high vigilance required in handling the aural polyps in pediatric population as early diagnosis and treatment are the key elements for successful outcomes.
\end{abstract}

\section{Keywords}

Embryonal Rhabdomyosarcoma, Aural Polyp, CSOM

\section{Introduction}

Aural polyps are well-circumscribed, soft, fleshy masses frequently found in the external auditory canal (EAC) of patients. They are usually inflammatory and suggest active middle ear disease presenting with ear discharge and hearing loss. Most commonly, polyps originate from middle ear mucosa and protrude into the external meatus through a tympanic membrane perforation [1]. The presence of a temporal bone malignancy must be consid-

How to cite this paper: Attakkil, A., Thorawade, V., Jagade, M., Kar, R., Rohe, D., Hanowate, R., Rangaraja, D. and Parelkar, K. (2015) Our Experience with Embryonal Rhabdomyosarcoma Presenting as Aural Polyp. International Journal of Otolaryngology and Head \& Neck Surgery, 4, 1-5. http://dx.doi.org/10.4236/ijohns.2015.41001 
ered, however, in any patient presenting with an aural polyp [1].

Rhabdomyosarcomas are highly aggressive locally destructive, malignant neoplasms of the soft tissue. The most common site involved by rhabdomyosarcoma is orbit followed by oral cavity and pharynx (29\%), the face and neck region (24\%) [2]; involvement of the ear and temporal bone with rhabdomyosarcoma is uncommon [3]. Rhabdomyosarcomas account for $5 \%$ to $15 \%$ or all childhood neoplasm and for $30 \%$ of temporal bone sarcomas. They are the most common malignant neoplasms of the temporal bone in childhood [4]. But the clinical presentation of this aggressive tumor mimics chronic suppurative otitis media which is a common clinical entity. The dilemma in diagnosis is often due to this close resemblance that results in the delay in diagnosis. Embryonal rhabdomyosarcoma of the middle ear is a rare and invariably fatal disease in children [5] distinct from other embryonal rhabdomyosarcomas of the head in its clinical presentation, prognosis and response to therapy. Early diagnosis and appropriate multimodality therapy holds key in the successful treatment of this rapidly growing tumor.

We present a case of embryonal rhabdomyosarcoma in a child which presented as polyp in the ear whose diagnosis was delayed in spite of the radiological and pathological evaluation. We intend to highlight the pitfalls in diagnosis that we are committed and hope that this case report may help the clinicians to attain an early and prompt diagnosis while dealing with these aggressive neoplasms. Our case report also reviews the histopathology, staging and treatment of embryonal rhabdomyosarcoma in brief.

\section{Case Report}

A 7 years old male child presented to a peripheral hospital with blood stained ear discharge and mass in the left ear which was accidentally noticed following a fall (Figure 1). He had no history of vertigo, decreased hearing or cranial nerve palsies at the time of presentation. Polypectomy was done and histopathological report showed granulation tissue consisting capillary sized vascular channels, mixed inflammatory infiltrate.

Following this the patient had recurrence of the polyp in the same ear with in twenty days. Computerised tomography (CT) scan of brain \& temporal bone was done which showed extensive soft tissue collection in the left external auditory canal, middle ear with rarefaction of the left petrous temporal bone suggestive of infective aetiology with bilateral otomastoiditis. Patient presented to our department with the same complaints. On examination, reddish polypoidal mass was filling the left external auditory canal with no mastoid tenderness. Tympanomastoid exploration of the left ear showed polypoidal tissue filling the antrum and middle ear which was removed in toto. Histopathological examination of the specimen done at two different centres reported nonspecific pyogenic inflammatory lesion of the left mastoid antrum. Patient developed left lateral rectus palsy 10 days following the surgery and ear discharge. MRI brain with CT temporal bone was done that showed ill defined contrast enhancement in petrous temporal bone on Left side with extension into infratemporal fossa suggestive of inflammatory/infective changes which was also seen in left EAC and middle ear cavity. There was

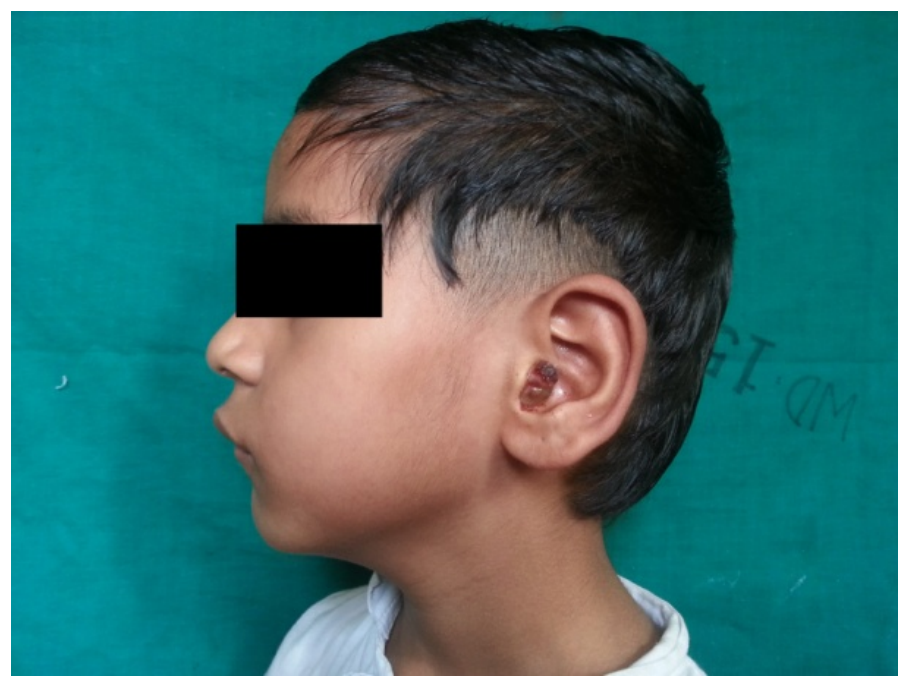

Figure 1. Showing the polypoidal mass protruding from the left EAC. 
also osteomyelitis of petrous bone. Neurologist advice was sought for and given osteomyelitis of temporal bone as provisional diagnosis; patient was started on intravenous Meropenem for 2 weeks according to pus culture and sensitivity report of the ear discharge. But the patient failed to show any improvement. Since there was osteopenia noted on the petrous temporal bone suggestive of tuberculosis, patient was started on anti Koch's treatment as advised by the paediatrician.

Patient's neurological signs worsened. He developed loss of general sensation over left half of forehead with deviation of angle of mouth with in next ten days. Meanwhile the recurrence of polypoidal tissue from left EAC was noted. He then developed House Brackmann grade four left lower motor neuron facial nerve palsy and left abductor palsy (Figure 2). MRI brain (plain + contrast) was repeated that showed inflammatory soft tissue in the left petrous temporal bone with extension into the left carotid canal, left meckel's cave, along the left sixth nerve and clivus, left cavernous sinus (Figure 3).

The biopsy of the mass was repeated and the histopathological examination showed the presence of strap cells showing rhabdomyoblastic differentiation, loosely arranged but with condensation beneath the epithelium (cambium layer) suggestive of embryonal rhabdomyosarcoma confirmed by immunohistochemistry (Figure 4). There was no CSF spread or distant metastasis. Patient was given chemo radiotherapy according to IRS-IV (Intergroup rhabdomyosarcoma study) protocol, falling into intermediate risk group. Patient was given induction chemotherapy with Vincristine + Ifosfamide + Etoposide + Mesna (VIE regimen) followed by radiotherapy and maintenance chemotherapy with Vincristine + Dactinomycin + Cyclophosphamide (VAC regimen). Total duration of chemoradiotherapy was 36 weeks. Patient tolerated the treatment well and is on follow up for last one year.

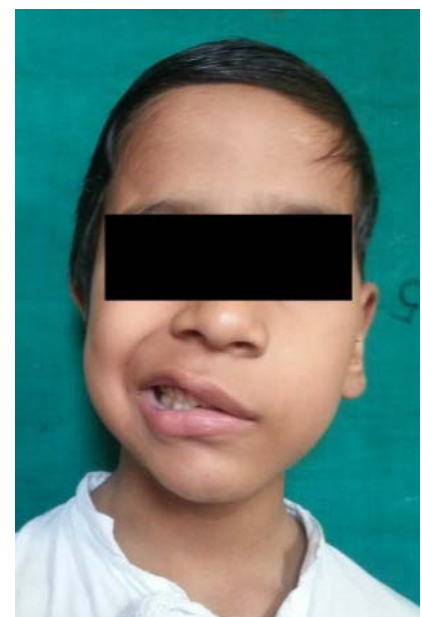

(a)

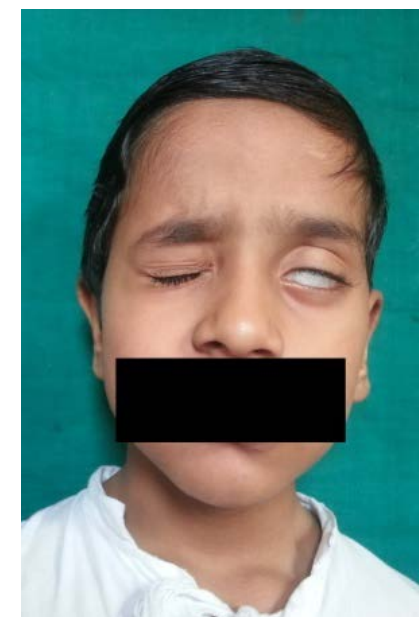

(b)

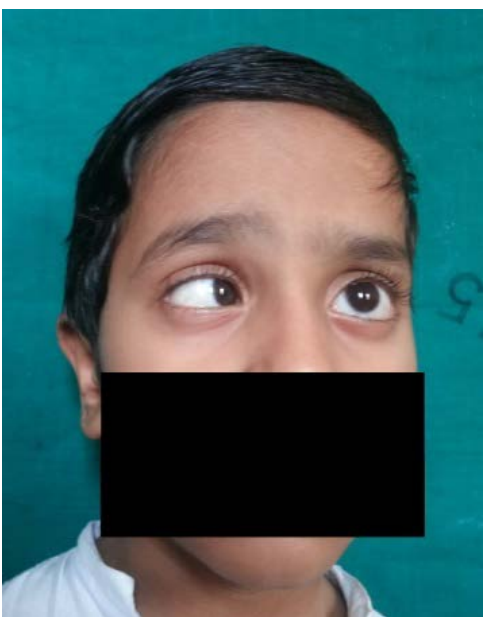

(c)

Figure 2. (a) (b) Showing left lower motor neuron facial palsy; (c) Showing left abductor palsy.
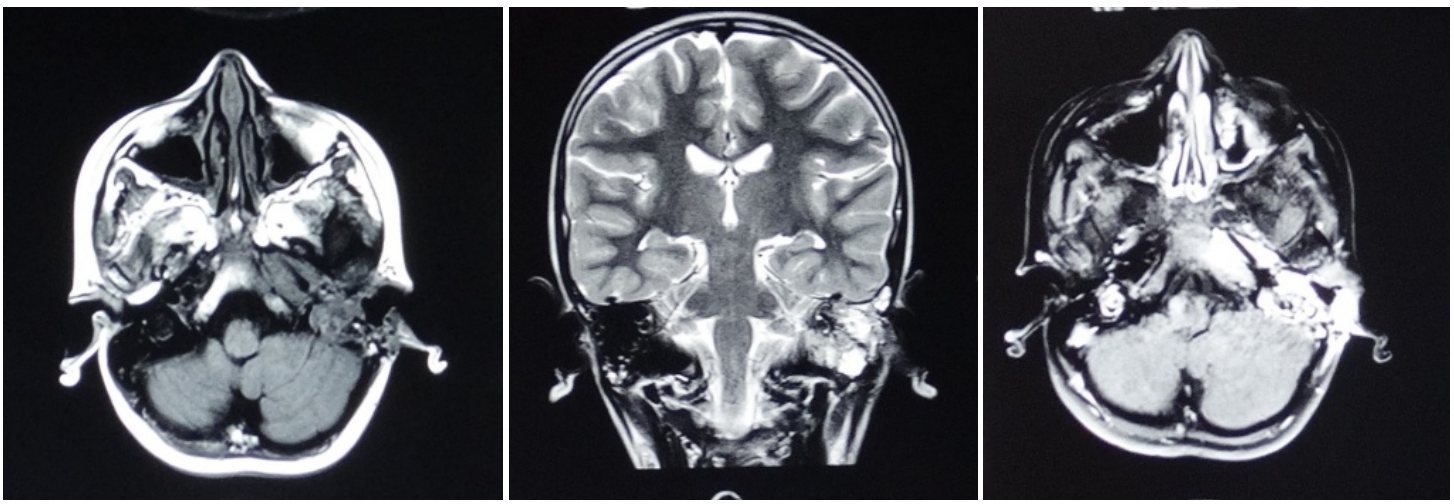

Figure 3. MRI Brain (plain + contrast) T1 \& T2 weighted images showing enhancing soft tissue in the left petrous temporal bone with extensions. 


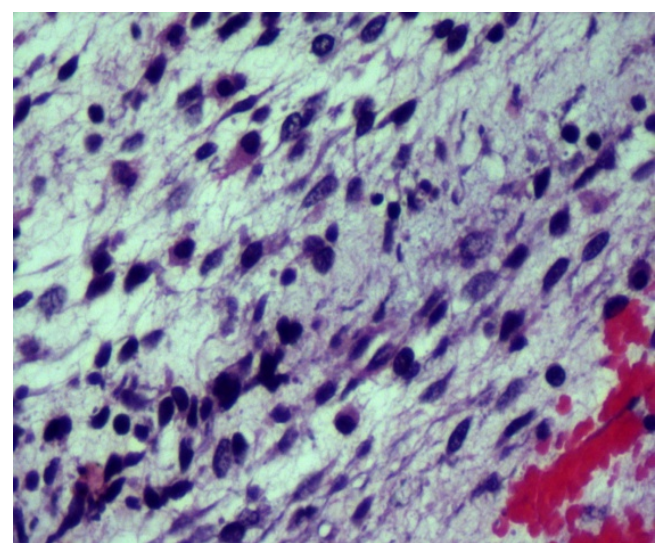

(a)

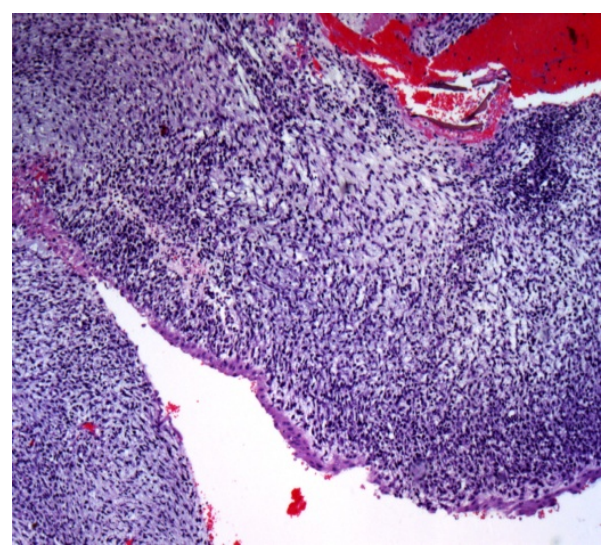

(b)

Figure 4. (a) Strap muscles showing rhabdomyoblastic differentiation; (b) Showing the arrangement of the cells beneath the cambium layer.

\section{Discussion}

Although comprising a large percentage of paediatric temporal bone tumours, temporal bone sarcomas are rare and represent less than $5 \%$ of all temporal bone malignancies with rhabdomyosarcoma the most common variety [1]. Forty percent of RMSs present in the head and neck. Commonly affected locations in the head and neck are the orbit, middle ear, oral cavity, nasopharynx and infratemporal fossa [4]. In the head and neck, rhabdomyosarcomas are divided into 3 categories: orbital (23\%), parameningeal (56\%) and nonparameningeal (21\%). Parameningeal denotes tumors that develop near the skull base and adjacent meninges [4].

The majority of the auricular rhabdomyosarcomas arise from the middle ear [1]. Only the embryonal subtype of rhabdomyosarcoma is recognized as the one occurring at the external auditory canal [6]. The majority of tumours present before the child are 12 years old, and the average age at presentation is 4.4 years [1]. Rhabdomyosarcomas are slightly more common in boys than in girls [7].

In the temporal bone, it is hypothesized that rhabdomyosarcomas originate from the malignant transformation of myocytes residing in the stapedial or tensor tympani muscles. Four histological subtypes have been described: embryonal, alveolar, pleomorphic and botryoid. In the head and neck, embryonal rhabdomyosarcomas are the most frequently encountered histological subtype (85\%) followed by alveolar rhabdomyosarcomas (15\%) [4].

The natural history of RMS in the temporal bone is aggressive local destruction with a propensity for distant metastases [1]. The primary presenting symptoms are similar to those of chronic otitis media: purulent and bloody otorrhea, otalgia, canal polyp and granulation tissue, and hearing loss [1]. As in our case patient presented with an ear polyp whose initial histopathological examination was inconclusive. In a case of recurrent ear polyp, the possibility of this aggressive tumour should always be tumour as the early detection makes the prognosis better. Only a high index of suspicion and clinical correlation will help the pathologist to identify the round cells usually admixed in the unspecific granulation tissue at an initial phase.

The diagnosis of rhabdomyosarcoma and the subtype is confirmed by histopathological examination. The characteristics of this polypoid tumour are those of rhabdomyoblasts and primitive mesenchymal cells showing a variable degree of skeletal muscle differentiation loosely arranged but with condensation beneath the epithelium (cambium layer) [6]. Embryonal rhabdomyosarcomas are so-named because of their remarkable evocation of developing skeletal muscle. As such they are characterized by variable zones of condensation that produce alternating foci of hypocellularity and hypercellularity. Like embryonic muscle, the dense zones typically contain areas of more overt myogenesis, whereas loose areas more closely resemble primitive mesenchyme and lie in a loose gelatinous matrix [8]. The immunohistochemistry markers desmin and myogenin yields clue to subclassification embryonal rhabdomyosarcomas usually stain in a more heterogeneous fashion with myogenin as shown in our case (Figure 5).

The staging of RMS depends on the type of RMS (embryonal or alveolar), the TNM stage and the clinical group. According to these three variables, they can be classified as low risk, intermediate risk, high risk groups whose 5 year survival rates are respectively $90 \%, 60 \%-80 \%, 20 \%-40 \%$ [7]. Our patient was diagnosed as an intermediate risk group and was given chemo radiotherapy. The types of treatment that can be used for RMS include 


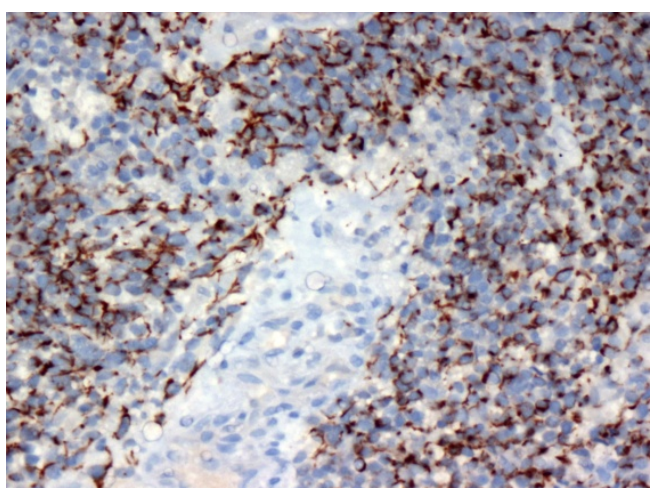

(a)

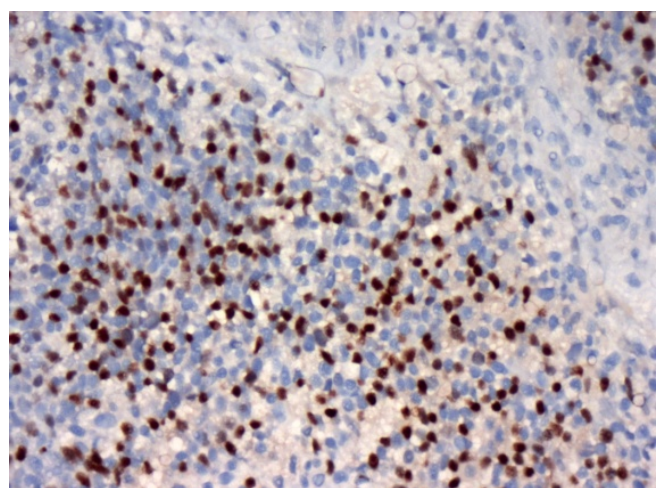

(b)

Figure 5. (a) The tumor cells showing immunoreactivity with desmin; (b) The tumor cells showing immunoreactivity with myogenin.

surgery, chemotherapy, radiation therapy or high-dose chemotherapy and stem cell transplant (very rarely). Chemotherapeutic regimen usually includes Vincristine + Ifosfamide + Etoposide + Mesna (VIE regimen) and Vincristine + Dactinomycin+ Cyclophosphamide (VAC regimen).

\section{Conclusion}

In this case report, we have described in detail the clinical course that patient went through and the delay in diagnosis that occurred in spite of the repeated radiological and histopathological evaluation. Though the temporal bone sarcomas are rare, only an approach with high clinical suspicion helps in diagnosing the disease at an early stage. We hope that this case report will guide the clinicians to approach the cases of recurrent ear polyp with high vigilance.

\section{References}

[1] Decker, B.C., Gulya, A.J. and Glasscock, M.E. (2003) Glasscock-Shambaugh Surgery of the Ear. Vol. 1, 5th Edition, PMPH, 353-755.

[2] Carol, J.M. and Richard, J.H. (2010) Pediatric Head and Neck Malignancies. In: Paul, W.F., Ed., Cummings Otolarymgology Head \& Neck Surgery, Vol. 3, Mosby, Philadelphia, 2835-2849.

[3] Chao, C.K., Sheen, T.S., Shau, W.Y., Ting, L.L. and Hsu, M.M. (1999) Treatment, Outcomes, and Prognostic Factors of Ear Cancer. Journal of the Formosan Medical Association, 98, 314-318.

[4] Bambakidis, N.C., Megerian, C.A. and Spetzler, R.F. (2009) Surgery of the Cerebellopontine Angle. PMPH USA Ltd., 261-262.

[5] Potter, G. (1966) Embryonal Rhabdomyosarcoma of the Middle Ear in Children. Cancer, 19, 221-226. http://dx.doi.org/10.1002/1097-0142(196602)19:2<221::AID-CNCR2820190213>3.0.CO;2-G

[6] Barnes, L. (2005) Pathology and Genetics of Head and Neck Tumours IARC. WHO Classification of Tumours Series, Volume 9 of World Health Organization Classification of Tumours, IARC Press, 335.

[7] http://www.cancer.org/acs/groups/cid/documents/webcontent/003136-pdf.pdf

[8] Parham, D.M. and Ellison, D.A. (2006) Rhabdomyosarcomas in Adults and Children. An Update. Archives of Pathology Laboratory Medicine, 130, 1454-1465 
Scientific Research Publishing (SCIRP) is one of the largest Open Access journal publishers. It is currently publishing more than 200 open access, online, peer-reviewed journals covering a wide range of academic disciplines. SCIRP serves the worldwide academic communities and contributes to the progress and application of science with its publication.

Other selected journals from SCIRP are listed as below. Submit your manuscript to us via either submit@scirp.org or Online Submission Portal.
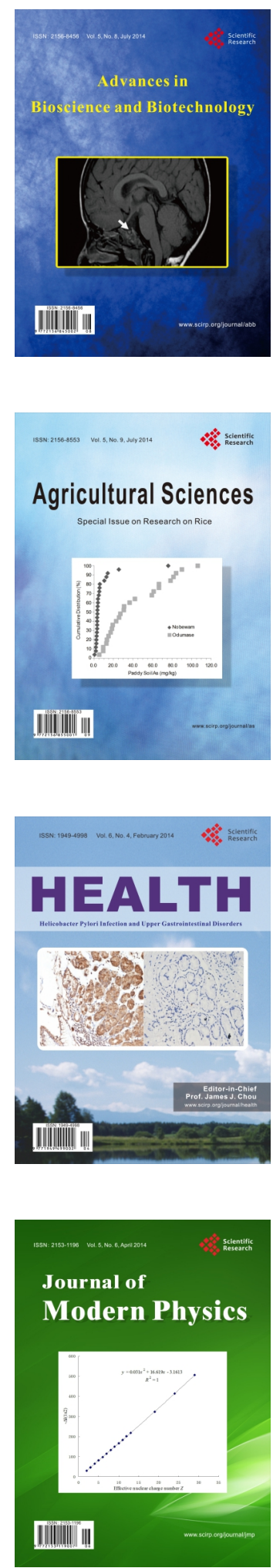
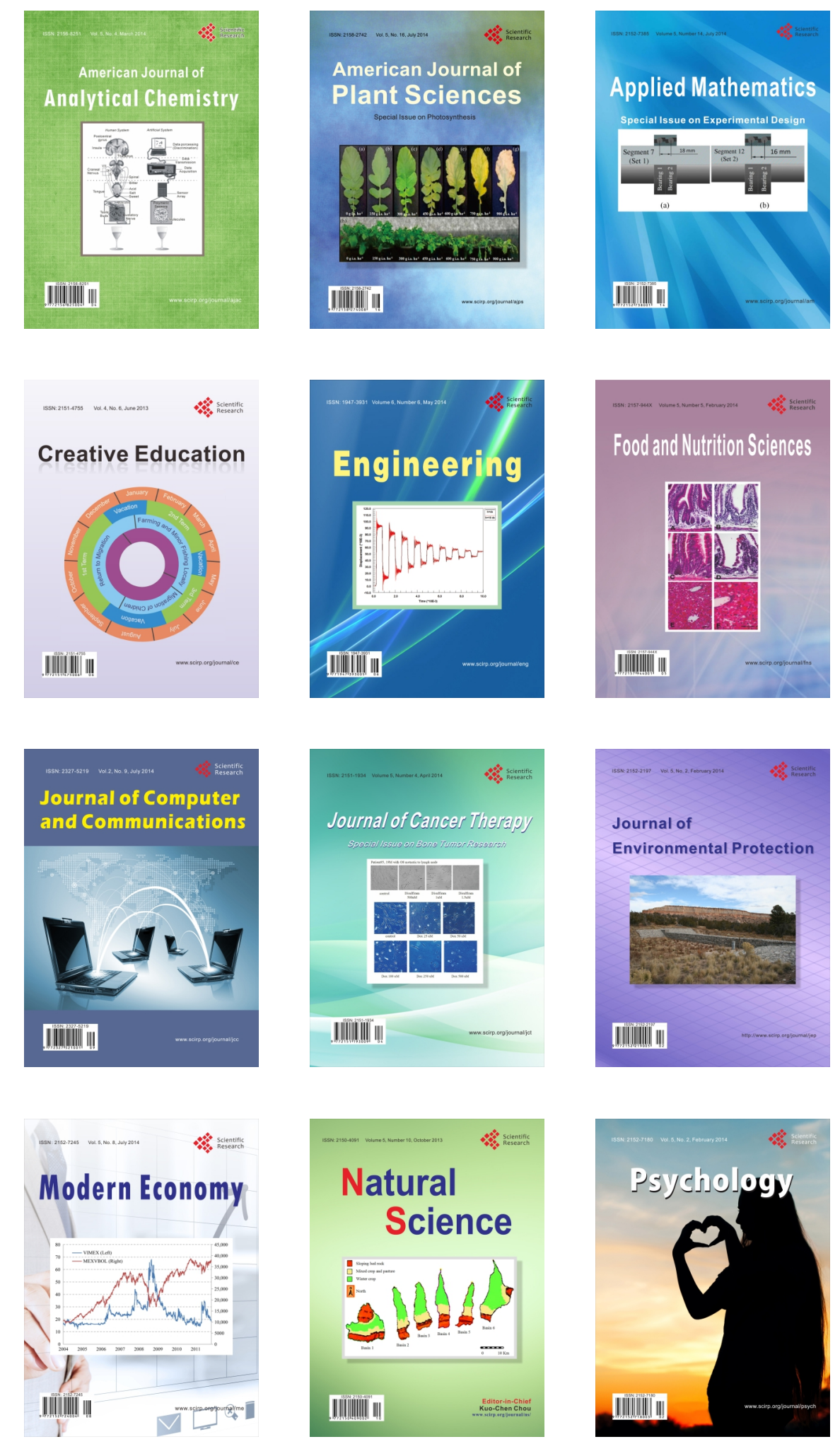